\title{
Perspektiven und Potenziale des Ausbildungs- und Berufsfeldes Altenpflege
}

\section{JOSEF HECKEN}

Staatssekretär im Bundesministerium für Familie, Senioren, Frauen und Jugend

\begin{abstract}
Gesellschaftliche Herausforderungen in Deutschland werden immer stärker durch den demografischen Wandel geprägt und wirken direkt auf die damit verknüpften Anforderungen an die Pflege. In keinem anderen Bereich werden die familiären, arbeitsmarktbezogenen und gesellschaftlichen Veränderungen so deutlich, wie in der Altenpflege. Dies stellt das Ausbildungs- und Berufsfeld der Altenpflege vor große Herausforderungen. Gleichzeitig liegen darin aber auch besondere Chancen, die die Politik und die Pflegebranche im Interesse der Pflegebedürftigen und der Pflegekräfte jetzt nutzen müssen.
\end{abstract}

\section{Pflege älterer Menschen - ein Ausbildungs- und Berufsfeld mit Zukunft}

Die Pflege älterer Menschen gehört heute zu den wichtigsten gesellschaftlichen Aufgaben. Die Herausforderungen werden besonders deutlich an den politischen Diskussionen über die Finanzierung der pflegerischen Leistungen sowie über die Qualitätssicherung und die Qualitätsprüfungen. Sie machen sich aber auch fest an den vielfältigen Weiterentwicklungen der Wohn- und Betreuungsformen, an der Stärkung des Verbraucherschutzes älterer pflegebedürftiger Menschen und nicht zuletzt an der besseren Unterstützung pflegebedürftiger Angehöriger.

Wenn es um die Fragen der besseren Gewährleistung einer qualitätsvollen und an den Bedürfnissen der Betroffenen orientierten Pflege in den unterschiedlichen Versorgungsformen geht, kommt aber eines der wichtigsten Handlungsfelder immer noch zu kurz: die notwendige Weiterentwicklung des
Ausbildungs- und Berufsfeldes der Altenpflege.

In der gesamtpolitischen Debatte über die Pflegepolitik blieb die Thematik lange zweitrangig. Umso bedeutender ist es, dass in den letzten Jahren hier eine dringend erforderliche Trendwende eingeleitet wurde.

Vieles ist derzeit auf Bundes- und Länderebene und in der Pflegebranche in Bewegung. Dies gilt sowohl für die Bildungspolitik in der Pflege als auch für den Beschäftigungsbereich der Altenpflege. Dabei wird der Stellenwert der Altenpflegerinnen und Altenpfleger als tragende Säule im Aufgabenfeld deutlich sichtbar. Sie verdienen - in all ihren unterschiedlichen Tätigkeitsbereichen - größte Hochachtung. Denn es geht um den täglichen professionellen und äußerst verantwortungsvollen Dienst an älteren Menschen, die schutz- und hilfebedürftig sind und die in unserer Gesellschaft Anspruch darauf haben, bestmöglich betreut und unterstützt zu werden. 


\section{Ausbildungspolitische Entwicklungen}

\subsection{Die Sicherung des Berufsnachwuchses - Altenpflegequalifizierung in föderalen Strukturen}

Unser Bildungssystem »Pflege « ist vielseitig, sehr ausdifferenziert und komplex. Wer heute nur die Unterscheidung nach Fachkraft und Hilfskraft vornimmt, wird den vielfältigen bildungspolitischen Entwicklungen, die die Altenpflege und die Pflege insgesamt in den letzten Jahren genommen haben, keinesfalls gerecht.

Auch in der politischen Diskussion ist eine differenziertere Befassung mit der Gesamtthematik dringend geboten. Erwartungen an Ausbildungsziele und Zugangsvoraussetzungen stehen teils im deutlichen Widerspruch zu den gleichzeitig erhobenen Ansprüchen an die pflegerische Versorgungsqualität.

Um die heutigen unterschiedlichen Qualifizierungsmöglichkeiten im Beschäftigungsfeld der Pflege älterer Menschen stärker anzuerkennen, um diese besser zu nutzen und um der Profession Pflege mehr gerecht zu werden, muss eine neue Balance zwischen der Bildungs-, Beschäftigungs- und der Fachpolitik in der Altenpflege geschaffen werden.

Voraussetzung dafür ist, dass die bildungspolitischen Entwicklungen sowohl auf Bundesebene als auch auf Länderebene stärker aufeinander bezogen werden und zu einem transparenten und allgemein anerkannten Stufensystem von Qualifizierungsmöglichkeiten führen. Jeder, der heute in der Altenpflege arbeiten möchte, kann bereits auf dem ihm angemessenen Qualifizierungsniveau einsteigen. Die entsprechenden Qualifizierungswege sind vorhanden, sind aber aufgrund der föderalen Strukturen oft nicht einfach zu überschauen.

So sind die Länder für die Qualifizierungsmaßnahmen im niedrigschwelligen Bereich sowie für die ein- bzw. zweijährigen Ausbildungen zu Pflegehelferinnen/Pflegehelfern bzw. zu Pflegeassistentinnen/Pflegeassistenten zuständig. In die Zuständigkeit des Bundes fallen die Regelungen über die Gesundheitsfachberufe, zu denen auch die Ausbildung zur Altenpflegerin/zum Altenpfleger gehört. Für den gesamten Fort- und
Weiterbildungsbereich sind hingegen wieder die Länder verantwortlich.

Unabhängig von diesen verschiedenen Qualifizierungsmöglichkeiten lässt sich aber eines klar festhalten: Entscheidend für die Sicherung des Berufsnachwuchses ist derzeit die Altenpflegeausbildung als dreijährige

Fachkraftausbildung. Sie ist der Dreh- und Angelpunkt im Bildungsbereich der Pflege älterer Menschen. Mit dem Altenpflegegesetz des Bundes hat sich ein bundeseinheitlicher und anspruchsvoller Ausbildungsstandard durchgesetzt. Zugleich hat sich auf dieser Grundlage in den vergangenen Jahren eine neue Ausbildungskultur entwickelt, die arbeitsmarktpolitisch, pflegepolitisch und gesellschaftlich von hohem Wert ist.

Das spiegelt sich vor allem in der positiven Entwicklung der Ausbildungszahlen wider. So befanden sich im Schuljahr 2010/2011 insgesamt rund 52.000 Schülerinnen und Schüler in dieser Berufsausbildung, davon rund 41.400 Frauen. Es handelt sich um den höchsten Wert, der in diesem Bildungsgang bisher erzielt wurde, und stellt allein eine Steigerung von rund $13 \%$ gegenüber dem Schuljahr 2009/2010 dar.

Es ist somit an der Zeit, von einem neuen Ausbildungstrend zu sprechen. Der Ausbildungsberuf der Altenpflegerin/des Altenpflegers nimmt - was kaum bekannt ist - einen der ersten Plätze unter allen bundesweit geregelten Ausbildungsberufen ein. Die vielfältigen Werbeaktionen und die Aufklärung über den Beruf und seine Perspektiven durch Bund, Länder, Verbände und Pflegeeinrichtungen scheinen zu greifen. Es ist angekommen, dass die Altenpflege sowohl Jugendlichen als auch lebenserfahrenen Menschen über den Weg der Umschulung eine sichere berufliche Zukunft eröffnet, attraktiv ist und vielfältige Entwicklungsmöglichkeiten bietet. In vielen Gesprächen mit Auszubildenden ist mir sehr deutlich geworden, dass hier eine selbstbewusste und von einem neuen beruflichen Selbstverständnis geprägte Generation von Pflegekräften, die sich bewusst für die Pflege alter Menschen entschieden hat, heranwächst.

Auf diesen positiven Entwicklungen dürfen wir uns aber nicht ausruhen. Um den Berufsnachwuchs für die kom- menden Jahrzehnte zu sichern, muss die Anzahl der Auszubildenden weiter kontinuierlich erhöht werden. Dies bedeutet, dass das Engagement aller Verantwortlichen sowohl im Bereich der Erstausbildung als auch der Umschulung nochmals verstärkt werden muss.

\section{intscheidend für die Sicherung des Berufsnachwuchses ist derzeit die Altenpflege- usbildung als dreijährige chkraftausbildung.}

Denn auch auf dem Ausbildungsmarkt zeichnen sich die demografischen Effekte ab. Durch die sinkende Zahl von Ausbildungsbewerberinnen und -bewerbern steigt der Konkurrenzdruck unter den Ausbildungsberufen. Hier wird sich die Altenpflege und die Pflege insgesamt behaupten müssen.

Die guten Ausbildungszahlen zeigen aber auch, dass sich die Pflegeeinrichtungen als Ausbildungsbetriebe etabliert haben und ihre diesbezügliche Verantwortung für die Sicherung des Berufsnachwuchses stärker wahrnehmen. Dazu gehört vor allem eine qualifizierte Ausbildung und eine enge Vernetzung mit den Altenpflegeschulen, die in der Berufsbildung oft als vorbildlich gilt. Von besonderer Bedeutung ist aber auch ein starkes Engagement für die Anwerbung von Jugendlichen. Nur wenn sich stationäre Pflegeeinrichtungen und ambulante Dienste jungen Menschen in der Berufsfindungsphase durch interessante Angebote öffnen und sich auf dem Ausbildungsmarkt als attraktive und aktive Ausbildungsstätten präsentieren, werden sie mit anderen Berufsausbildungen konkurrieren können.

Die Aufgabe, noch mehr Jugendliche für die Altenpflegeausbildung zu gewinnen, kann aber nur dann erfolgreich sein, wenn auch bestimmte Zielgruppen stärker angesprochen werden. So wird es darauf ankommen, mehr männliche Jugendliche zu gewinnen. Besondere Initiativen, wie der bundesweite Boys'

\footnotetext{
1 In der Statistik des Statistischen Bundesamtes »Schüler/innen in Sozial- und Gesundheitsdienstberufen (insgesamt und 1. Schuljahrgang) $2010 / 2011$ «sind Zahlen für Hessen, Bremen und Hamburg nicht enthalten.
} 
Day, bilden hier wichtige Ansätze, die auf allen Verantwortungsebenen ausgebaut werden müssen.

Auch sind verstärkte Anstrengungen erforderlich, um mehr junge Migrantinnen und Migranten auf die Ausbildung aufmerksam zu machen. Unabhängig davon, dass sie einen zukunftssicheren Arbeitsplatz finden, werden sie aufgrund ihrer besonderen Qualifikation im Bereich der kultursensiblen Pflege dringend gebraucht.

Entscheidend für die Anwerbung von Jugendlichen wird auch in Zukunft sein, ob die Ausbildung modern und attraktiv ist. Auf Bundes- und Länderebene sind vielfältige Projekte, die diesen Zielen dienen, auf den Weg gebracht worden. Für das Bundesministerium für Familie, Senioren, Frauen und Jugend möchte ich beispielhaft auf das Handbuch für eine kultursensible Altenpflegeausbildung, auf das Projekt »Servicenetzwerk Altenpflegeausbildung «, in dem drei Jahre lang Pflegeeinrichtungen in allen Fragen der Altenpflegeausbildung beraten wurden sowie auf die vielfältigen Informationsmaterialien zu Ausbildung und Beruf hinweisen. Ganz aktuell ist das Vorhaben »Modell einer gestuften und modularisierten Altenpflegequalifizierung «. Es wurde im November 2011 abgeschlossen. In diesem Projekt wurden u. a. Konzepte entwickelt und erprobt, um die Ausbildungsgänge in der Altenpflege (Helfer-, Fachkraftausbildung, Weiterbildungslehrgänge) zu modularisieren. Dieses Vorhaben ist mir sehr wichtig, denn modularisierte Bildungsgänge fördern die Durchlässigkeit, ermöglichen den Erwerb von Teilqualifikationen, erleichtern die Anrechnung von Kompetenzen, die an anderer Stelle erworben wurden, und ermöglichen eine schnellere Anpassung der Ausbildungsinhalte an neue berufliche Entwicklungen. Dies sichert Qualität und schafft Attraktivität.

Ein Hemmnis vor allem für kleinere stationäre Pflegeeinrichtungen und für ambulante Dienste stellt immer wieder die Finanzierung der Ausbildung dar. Es ist deshalb sehr zu begrüßen, dass neben Baden-Württemberg und RheinlandPfalz jetzt auch Nordrhein-Westfalen und das Saarland von der gesetzlichen Möglichkeit Gebrauch gemacht haben, ein Umlageverfahren zur Aufbringung der Kosten der Ausbildungsvergütung einzuführen. So werden alle stationären und ambulanten Pflegeeinrichtungen unabhängig davon, ob sie selbst ausbilden - in die Finanzierung eingebunden.

\subsection{Potenziale und Perspektiven des Ausbildungsbereichs}

Um den Personalbedarf auf Dauer decken zu können, die Qualität zu sichern und das Berufsfeld Pflege auch zukünftig attraktiv zu halten, müssen die Möglichkeiten, die die Ausbildung, aber auch die Nachqualifizierung sowie die Fort- und Weiterbildungen bieten, ausgebaut werden. Wichtig ist in diesem Zusammenhang, die Durchlässigkeit zwischen den Bildungsgängen weiter $\mathrm{zu}$ verbessern. Dieses ist aufgrund der unterschiedlichen Zuständigkeiten von Bund und Ländern eine besondere Herausforderung. Aber es gehört zu den politischen Aufgaben auf allen Ebenen, diesen Prozess in den kommenden Jahren stärker zu steuern und zu stützen.

Gleiches gilt für das besondere politische Ziel, das Potenzial, das in den Pflegeausbildungen insgesamt steckt, in Zukunft noch besser auszuschöpfen. Die Bundesregierung hat sich hier grundlegende neue Weichenstellungen vorgenommen. Die drei Pflegeberufe Altenpflege, Gesundheits- und Krankenpflege sowie Gesundheits- und Kinderkrankenpflege sollen zu einem neuen Pflegeberuf zusammengeführt werden.

Für mich ist dies aus der Perspektive der Altenpflege ein wichtiger, notwendiger und erfolgversprechender Weg. Die Altenpflege war und ist als Beruf immer im Auf- und Umbruch. Gerade in bewegten Zeiten kann und darf es keinen Stillstand geben. Jetzt geht es darum, das Kompetenzprofil noch breiter aufzustellen. Es geht nicht um eine Zäsur; denn die Zukunft der Pflege liegt in der Pflege älterer Menschen. Es geht vielmehr um eine konsequente und gemeinsame Weiterentwicklung der Pflegeausbildungen. Und weil dies so ist, stehen auch alle Innovationen und Aktivitäten zur Etablierung einer guten Ausbildung in den Pflegeeinrichtungen dazu nicht im Widerspruch. Vielmehr werden sich die Vorreiterrollen auch in Zukunft auszahlen.

Die Zusammenführung ist aus meiner Sicht vor allem deshalb wichtig, weil die heutige Differenzierung der
Pflegeberufe den Entwicklungen der Versorgungsstrukturen und den Anforderungen an die professionelle Pflege der Zukunft nicht mehr entsprechen werden. Neue Potenziale und Perspektiven werden sich dann ergeben, wenn die formale Trennung der drei Berufe überwunden wird. Es ist wichtig, dass die Pflegefachkräfte universeller und flexibler eingesetzt werden können, dass sie in der Ausbildung ein möglichst breites Qualifikationsprofil erwerben. Bereits heute wäre es sehr wichtig, dass die Krankenhäuser mehr Altenpflegerinnen und -pfleger einstellen. Schon lange sind Gesundheits- und Krankenpfleger/ innen in den stationären Pflegeeinrichtungen beschäftigt. In den ambulanten Diensten sind beide Berufsgruppen präsent. Sowohl die heutigen Berufsgesetze als auch die Praxis belegen nicht zuletzt, dass die Pflegefachkräfte sich in bestimmten Qualifikationsbereichen überschneiden und in vielfach dieselben Aufgaben wahrnehmen.

Ich bin davon überzeugt, dass eine Zusammenführung der Pflegeausbildungen auch die Attraktivität des Berufsfeldes erhöhen wird, weil sich für die Absolventinnen und Absolventen mehr berufliche Entwicklungsmöglichkeiten ergeben. Letztlich geht es um einen Berufsbereich, der sich stetig weiterentwickelt, so dass die Stichworte »spätere Spezialisierung, Weiterbildung, lebenslanges Lernen" das Berufsfeld auch in Zukunft prägen werden.

\section{Die Altenpflege war und ist als Beruf immer im Auf- und Umbruch.}

Wir gehen den neuen Weg nach intensiven Vorarbeiten durch Fachpolitik, Wissenschaft und Praxis. Vielfältige Projekte wurden durchgeführt. Das Bundesministerium für Familie, Senioren, Frauen und Jugend hat in der letzten Legislaturperiode das große Modellvorhaben »Pflegeausbildung in Bewegung « umgesetzt und wissenschaftlich evaluieren lassen. Länder und Verbände haben sich positioniert und überwiegend für die Zusammenführung ausgesprochen. Gegenwärtig entwickelt eine auf Fachebene eingesetzte Bund-LänderArbeitsgruppe Eckpunkte, die Anfang 2012 vorgelegt werden sollen. Auf dieser 
Grundlage wird die Bundesregierung in diesen Jahr über die konkrete Ausgestaltung eines neuen Berufsgesetzes politisch entscheiden und einen Gesetzentwurf vorlegen.

$\mathrm{Zu}$ berücksichtigen ist dabei aber noch eine aktuelle Entwicklung auf EU Ebene. So hat die EU-Kommission sehr kurzfristig einen Vorschlag zur Änderung der EU-Berufsanerkennungsrichtlinie vorgelegt, die die nationale Gesetzgebungsarbeit im Bereich der Pflegeberufe beeinflussen wird. Die EU-Kommission plant, die Zugangsvoraussetzung der Ausbildungen für die allgemeine Pflege in Europa von bisher 10 auf 12 Jahre allgemeine Schulbildung anzuheben. Die Bundesregierung und die Länder haben sich bereits gegen diese Änderung aus gesprochen, um eine Verschärfung des Fachkräftemangels zu vermeiden.

Da noch nicht abzusehen ist, wie in Brüssel entschieden wird, stehen wir - auch bezogen auf die nationale Gesetzgebung - noch vor spannenden Diskussionen, die vor allem auch die Entwicklung der Qualifizierung auf akademischer Ebene einschließt. Hier ist bereits einiges in Bewegung. Gegenwärtig werden in den Bundesländern unterschiedliche Modelle erprobt. Ich begrüße diese Entwicklung. Für mich steht aber fest, dass auch in Zukunft die dreijährig an den Berufsfachschulen ausgebildeten Pflegefachkräfte die wichtigste und stärkste Säule im Berufsfeld bleiben müssen. Einen Bedarf an akademisch ausgebildeten Pflegekräften wird es ganz sicher geben. Dieser Berufsbereich muss zusätzlich aufgebaut werden. Er kann und darf aus meiner Sicht die berufliche Ausbildung jedoch nicht ersetzen.

Weitreichende Veränderungen in der Ausbildungslandschaft liegen also vor uns. Dies ist aber noch nicht alles. Vielmehr ist es ein weiteres wichtiges Ziel der Bundesregierung, das Kompetenzprofil der Pflegefachkräfte durch die Übertragung heilkundlicher Tätigkeiten zu erweitern. Der Gemeinsame Bundesausschuss hat im Oktober 2011 die Richtlinie zur Heilkundeübertragung im Rahmen von Modellvorhaben vorgelegt. In den kommenden Monaten wird das BMFSFJ dazu auf der Grundlage der Modellklausel im Altenpflegegesetz Projekte planen und durchführen. Denn ich halte es für sehr wichtig, dass heilkundliche Aufgaben von Altenpflegerin- nen und -pflegern eigenverantwortlich übernommen werden - ein Meilenstein in der Geschichte der Altenpflege.

Beachtenswert sind natürlich auch die vielfältigen Entwicklungsprozesse im Bereich der Assistenzausbildungen und der Weiterbildungsgänge auf Länderebene.

All diese Entwicklungen zeigen, dass die Pflege sich als moderne Bildungslandschaft etabliert, die auf Durchlässigkeit, Transparenz und Flexibilität ausgerichtet ist.

\subsection{Die Entwicklungen des Berufsfeldes}

3. Das Berufsfeld der Altenpflege

Der Beschäftigungsbereich der Altenpflege entwickelt sich sehr dynamisch. Waren im Jahr 1999 noch 630.000 Mitarbeiterinnen und Mitarbeiter in ambulanten und stationären Pflegeeinrichtungen beschäftigt, sind es heute bereits 890.000 . Das ist eine Steigerung von ca. $41 \%$ in nur 10 Jahren.

Das Beschäftigungsfeld der Altenpflege ist mittlerweile sehr breit aufgestellt. Zahlreiche Berufsgruppen sind vertreten. Darüber hinaus findet eine Vielzahl einjährig ausgebildeter Pflegehelfer/ innen und angelernter Hilfskräfte hier eine Beschäftigung. Eine Schlüsselrolle kommt natürlich den Pflegefachkräften, und hier vor allem den Altenpflegerinnen und Altenpflegern zu. Gegenüber nahezu 110.000 Fachkräften im Jahr 1999 sind heute rund 195.000 Altenpflegerinnen und Altenpfleger in 11.600 stationären Pflegeeinrichtungen und 12.000 ambulanten Diensten tätig. ${ }^{2}$ Ihre Zahl hat sich also innerhalb von 10 Jahren nahezu verdoppelt.

Zugleich stehen wir aber vor der Herausforderung, den steigenden Bedarf an Pflegefachkräften weiterhin zu decken. Vielerorts besteht schon ein Fachkräftemangel. Zahlen der Bundesagentur für Arbeit verdeutlichen dies. So waren bereits im Frühjahr letzten Jahres 9.200 offene Stellen gemeldet. Arbeitslos gemeldet waren zum gleichen Zeitpunkt rund 3.500 Altenpflegerinnen und Altenpfleger.

Nach wie vor ist die Altenpflege ein von Frauen geprägter Beruf. Mehr als $85 \%$ der Beschäftigten in der Altenpfle- ge sind weiblich. Im Vergleich zu anderen Branchen zeichnet sich die Altenpflege durch eine sehr hohe Teilzeitquote aus. So liegt der Anteil der in Teilzeit beschäftigten Altenpflegerinnen und Altenpflegern 
die erforderlichen Anstöße für innovative Weiterentwicklungen geben und Einrichtungen darin unterstützen, das Personalmanagement zukunftsgerecht auszurichten. Beispielhaft verweise ich auf die »Initiative Neue Qualität der Arbeit « (INQA) und auf die Gemeinsame Deutsche Arbeitsschutzstrategie.

Wie wichtig es ist, dass Politik und Pflegeeinrichtungen den besonderen Fokus auf die Pflegekräfte, auf das Berufsfeld der Altenpflege richten, unterstreicht nicht zuletzt auch die erst kürzlich von der Universität Witten/Herdecke durchgeführte Umfrage für den »Gesundheitsmonitor 2011«. Danach wählen Pflegebedürftige ihre Pflegeeinrichtung vor allem nach der Qualität des Personals aus. So sei entscheidend, ob ausreichend Pflegekräfte vorhanden seien, ob sie freundlich und zugewendet seien, über welche Ausbildung sie verfügten, ob sie respektvoll mit den Bewohnerinnen und Bewohnern umgingen und wie viel Ziel sie hätten.

Es ist gleichsam bekannt, dass viele Pflegefachkräfte diesen Anforderungen, aber auch den selbst gesetzten Ansprüchen nicht immer gerecht werden können. Die meisten Altenpflegerinnen und Altenpfleger fühlen sich dem Beruf eng verbunden und möchten möglichst lange in der Altenpflege arbeiten. Die Berufsverläufe zeigen aber vielfältige Unterbrechungszeiten, die unter anderem auf schwierige Arbeitsbedingungen zurückgeführt werden. Auch höre ich von Pflegefachkräften, dass sie ihr Wissen und Können nicht immer einsetzen, weil ihnen die Zeit dafür fehlt.

Ich sehe hier großen Handlungsbedarf. Pflegebranche und Politik sind auf allen Verantwortungsebenen gefordert, dazu beizutragen, dass die beruflichen Rahmenbedingungen für Pflegekräfte weiter verbessert werden.

Dazu gehört auch, dass die Pflegeeinrichtungen stärker auf Arbeitsteilung setzen, um den Aufgaben in dem vielseitigen Beschäftigungsfeld mit unterschiedlichen Verantwortungsbereichen besser gerecht zu werden und den Personaleinsatz unter qualitativen Gesichtspunkten effizienter zu planen.

Um hier neue Wege aufzuzeigen, wurde in dem bereits oben genannten Projekt »Modell einer gestuften und modularisierten Altenpflegequalifizierung « der »Entwurf eines Qualifikationsrah- mens für den Beschäftigungsbereich der Pflege, Unterstützung und Betreuung älterer Menschen « entwickelt, der jetzt in Pflegeeinrichtungen erprobt werden soll. Es handelt sich um ein neues Koordinatensystem für die Pflegebildung und für das Beschäftigungsfeld der Altenpflege, das die Anforderungs- und Qualifikationsprofile in gestufter, anschlussfähiger Form - von den niedrigschwelligen Hilfen bis zur Hochschulqualifizierung - beschreibt.

Entscheidend ist aber auch, dass die Probleme der Pflegefachkräfte sehr ernst genommen werden. Als oberste Maxime muss gelten: Pflegekräfte müssen ihren Beruf adäquat ausüben und ihre beste Leistung abrufen können. Wir müssen vor allem gute Lösungen finden, um dem immer wieder kritisierten erheblichen Zeitdruck zu begegnen.

Dies ist im Interesse der Pflegebedürftigen, aber auch der Pflegebranche insgesamt. Denn von der Zufriedenheit der Mitarbeiterinnen und Mitarbeiter hängt entscheidend $\mathrm{ab}$, ob sie der Pflege treu bleiben. Die Schaffung guter Arbeitsbedingungen ist somit eine der wichtigsten Stellschrauben für die Deckung des Fachkräftebedarfs.

\section{Die Schaffung guter Arbeitsbedingungen ist somit eine der wichtigsten Stellschrauben für die Deckung des Fachkräftebedarfs.}

Hinzukommen muss aber auch, dass das Fachkräftepotenzial durch ein größeres Angebot an Vollzeitstellen stärker erschlossen wird. Wenn heute mehr als die Hälfte der Fachkräfte in Teilzeit arbeitet, geht für das Beschäftigungsfeld viel Potenzial verloren. Dies wird sich die Pflegebranche auf Dauer nicht leisten können. Mir ist bewusst, dass die Altenpflege durch die Flexibilität der Arbeitszeiten insbesondere für viele Frauen attraktiv ist. Dies soll auch so bleiben. Zugleich wird es aber gerade in der Pflegebranche darauf ankommen, die Angebote zur Vereinbarkeit von Familie und Beruf deutlich zu verbessern, damit auch die Vollzeitbeschäftigung für Pflegekräfte möglich wird. Hier sehe ich noch erheblichen Nachholbedarf.

Wenn über die Potenziale und Pers- pektiven im Beschäftigungsfeld nachgedacht wird, darf nicht unberücksichtigt bleiben, dass nach Deutschland Zugewanderte in anderen Ländern eine berufliche Qualifikation erlangt haben, die auf dem deutschen Arbeitsmarkt nicht ausreichend genutzt werden kann. Für die Sicherung der Fachkräftebasis ist es deshalb auch wichtig, alle Qualifikationspotenziale im Inland zu aktivieren, zu nutzen und Deutschland für qualifizierte Zuwanderung attraktiver zu machen. Einen wesentlichen Beitrag dazu wird das Gesetz zur Verbesserung der Feststellung und Anerkennung im Ausland erworbener Berufsqualifikationen " (sog. »Anerkennungsgesetz«) leisten, das im April 2012 in Kraft treten wird.

Für die Altenpflege ergibt sich insbesondere eine neue Entwicklung daraus, dass für die Beurteilung von Berufsqualifikationen aus Drittstaaten (also nicht EU) eine nahezu vollständige Angleichung an die Verfahren für die Beurteilung von Berufsqualifikationen aus EUMitgliedstaaten erfolgt. Wesentlicher Maßstab bei der Anerkennung aller ausländischen Berufsabschlüsse ist fortan ihr Inhalt, ihre Qualität und die Gleichwertigkeit zur Altenpflegeausbildung, wobei Berufserfahrung berücksichtigt wird. Liegt keine Gleichwertigkeit zur Altenpflegeausbildung vor, haben die Antragstellerinnen und Antragsteller Ausgleichsmaßnahmen (Anpassungslehrgang oder Prüfung) zu absolvieren.

Nicht zuletzt gilt es zu berücksichtigen, dass mit Eintritt der uneingeschränkten Freizügigkeit für Arbeitnehmerinnen und Arbeitnehmer aus den acht EU-Mitgliedstaaten Polen, Litauen, Lettland, Estland, Tschechische Republik, Slowakei, Ungarn und Slowenien Anfang Mai 2011 die Arbeitserlaubnispflicht und die Vorrangprüfung für Pflegefachkräfte entfallen sind. Die Freizügigkeit eröffnet grundsätzlich den Weg, ausländische Pflegefachkräfte für eine Beschäftigung in Deutschland zu gewinnen. Bereits jetzt zeichnet sich jedoch ab, dass die Fachkräfte aus den o.g. Ländern auch in ihren Heimatländern verstärkt gebraucht werden bzw. bereits in andere Mitgliedstaaten ausgewandert sind. Es bleibt abzuwarten, inwieweit sich die uneingeschränkte Freizügigkeit positiv auf die Pflegefachkräftesituation in Deutschland auswirken wird. 


\section{Verantwortung für morgen übernehmen}

Damit die Altenpflege auch in Zukunft ihrer hohen Verantwortung gerecht wird, sind innovative Weiterentwicklungen erforderlich. Dies gilt sowohl für die Pflegebildungslandschaft, für den Arbeitsmarkt Pflege und für die Pflegeunternehmen. Nur wenn hier wir auf breiter Ebene Verbesserungen erreichen, werden sich dauerhaft ausreichend junge, aber auch lebenserfahrene Menschen für die Altenpflege entscheiden und dort lange ihren Beruf ausüben.

Es ist entscheidend, den bereits eingeleiteten Wandlungsprozess im Berufsund Beschäftigungsfeld der Altenpflege jetzt durch ein umfassendes Maßnah-

\section{Pflegebildung muss dem Wandel in der Pflege Rechnung tragen!}

menpaket noch konsequenter und wirkungsvoller voranzubringen. Dies gelingt meines Erachtens nur, wenn sich alle Verantwortlichen in der Altenpflege zusammenschließen, ihre Kräfte bündeln und die notwendigen Maßnahmen kurzfristig umsetzen.

Den Worten folgen Taten: Die Bundesregierung hat unter Federführung des Bundesministeriums für Familie, Senioren, Frauen und Jugend gemeinsam mit den Ländern und den Verbänden eine »Ausbildungs- und Qualifizierungsoffensive Altenpflege" gestartet. Ziel dieser wichtigen und in der Altenpflege einmaligen Initiative ist es, die Anstrengungen zur Sicherung des Berufsnachwuchses und zur Verbesserung der Fachkräftesituation in der Altenpflege zu verstärken, gemeinsame Ziele zu formulieren und Vereinbarungen $\mathrm{zu}$ treffen, die bis zum Jahre 2014 von den Partnern umgesetzt werden sollen. Hierzu wurde zunächst eine Arbeitsgruppe eingerichtet, die zu zehn Handlungsfeldern Zielvereinbarungen vorbereitet. Anfang 2012 soll die Vereinbarung auf Spitzenebene unterzeichnet werden. Im Rahmen dieser Offensive geht es um die Erhöhung der Zahl der Altenpflegeauszubildenden. Es sollen Maßnahmen zur besseren Ausschöpfung des Nachqualifizierungspotenzials und der Vereinbarkeit von Familie und Beruf bzw. Familie und Ausbildung ergriffen werden. Aber auch Themen wie »Umschulung «, »Gesellschaftliche Bedeutung des Berufsfeldes Altenpflege «, "Attraktive Beschäftigungsbedingungen in der Altenpflege « und »Arbeitnehmerfreizügigkeit« sollen im Rahmen der Ausbildungs- und Qualifizierungsoffensive eine wichtige Rolle spielen und zu konkreten Fortschritten führen.

\section{Fazit}

Für die Herausforderungen, vor die uns der demografische Wandel stellt, gilt insgesamt: Alle Verantwortlichen in der Pflege sind aufgefordert, sich an einer modernen Weiterentwicklung der Altenpflege als Ausbildungs- und Berufsfeld zu beteiligen. Denn: Wir können uns auf diesem Feld keine wesentlichen Verzögerungen leisten. Pflegebildung muss dem Wandel in der Pflege Rechnung tragen! Die Beschäftigungsbedingungen müssen attraktiver werden, damit sich nach wir vor viele Menschen dauerhaft für dieses wichtige Berufsfeld der Zukunft entscheiden.

\section{Altenpflege am Scheideweg}

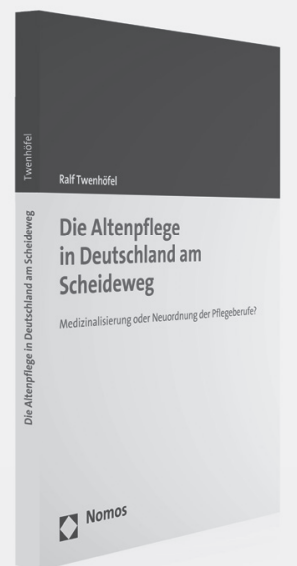

\author{
Die Altenpflege \\ in Deutschland \\ am Scheideweg \\ Medizinalisierung \\ oder Neuordnung \\ der Pflegeberufe? \\ Von Prof. Dr. Ralf Twenhöfel \\ 2011, 176 S., brosch., 29,-€ \\ ISBN 978-3-8329-6037-5
}

"Ein sehr wichtiges, interessantes und nachdenklich machendes Buch... eine hervorragende Studie, die als Standardlektüre in meinen Veranstaltungen eingesetzt wird."

Prof. Dr. Hermann Brandenburg, www.socialnet.de April 2011 www.nomos-shop.de/12996

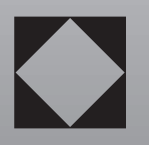

